\title{
Original
}

\section{Comparison of a Hydroxyapatite-Coated and an Anodic Oxidized Titanium Implant for Experimentally Induced Peri-Implantitis: Macroscopic and Novel Radiographic Evaluations in a Canine Model}

\author{
Hiroki Nakamura'), Tomonori Matsuno(1), Yoshiya Hashimoto's), Tatsuo Nakamura ${ }^{3)}$ and Izumi Mataga() \\ 1) Department of Oral \& Maxillofacial Surgery, The Nippon Dental University School of Life Dentistry at Tokyo, Tokyo, Japan \\ ${ }^{2)}$ Department of Biomaterials, Osaka Dental University, Osaka, Japan \\ ${ }^{3)}$ Department of Bioartificial Organs, Institute for Frontier Medical Sciences, Kyoto University, Kyoto, Japan \\ (Accepted for publication, September 3, 2015)
}

\begin{abstract}
This study aimed to compare the response of peri-implant tissue surrounding hydroxyapatite-coated titanium (HA) implants and anodic oxidized titanium (AnO) implants, as well as associated ligature-induced peri-implantitis. The third and fourth premolars (P3, P4) of the mandible were extracted in five beagle dogs. After 2 months, 10 dental implants with different surfaces (HA/AnO) were inserted into the mandible in five beagle dogs. A plaque control program was maintained until the second-stage surgery. Three months after implantation, a healing-cap connection was performed, and silk ligatures were placed in the submarginal position around the neck of the implants to induce peri-implantitis. Macroscopic evaluations including modified plaque index (mPI), modified gingival index (mGI), and probing depth (PD), and radiographic evaluations including dental radiography and multislice computed tomography (MSCT) were conducted for 24 weeks following ligature placement. Bone loss around the implant was evaluated according to the radiolucent area on dental radiographic images and the hyperpermeability area on MSCT images. All macroscopic parameters except PD exhibited a consistent increase for all implants. Over the course of the study, bone loss associated with the HA implant was greater than losses with the AnO implant. Following evaluation of dental radiographs at 20 and 24 weeks after ligature placement, a significant difference was detected between the HA and AnO implants $(\mathrm{P}<$ 0.05). The results suggested that the resistance of the HA implant to peri-implantitis was inferior to that of the AnO implant.
\end{abstract}

Key words: Peri-implantitis, Hydroxyapatite-coated, Anodic oxidized, Dental radiography, Multislice computed tomography

\section{Introduction}

Dental implant therapy has emerged as a highly successful treatment for the replacement of lost teeth. Further, implant survival rates were reported to be greater than $89 \%$ at $10-15$ years following performance of corrective procedures ${ }^{1-3}$. However, supportive bone around dental implants can be lost, even in cases of successful osseointegration.

Numerous potential causes of implant failure exist, but most researchers agree that peri-implant infection leads to clinical failure in all implant types. During the 6th European Workshop on Periodontology in 2008, it was proposed that the term "peri-implant disease" be used as a "collective term for inflammatory reactions in the tissue surrounding an implant," while peri-implant mucositis be used to describe "the presence of inflammation in the mucosa

Correspondence to: Dr. Hiroki Nakamura, Department of Oral \& Maxillofacial Surgery, The Nippon Dental University School of Life Dentistry at Tokyo, 1-9-20 Fujimi, Chiyoda-ku, Tokyo, 102-8159 Japan; Te1: +81-3-3261-6558; Fax: +81-3-3512-0571; E-mail: tombow0114@gmail.com at an implant with no signs of loss of supporting bone." Additionally, it was proposed that the term "peri-implantitis" be used to describe an "inflammatory process around an implant, characterized by soft tissue inflammation and loss of supporting bone"4). Following 10 years of post-treatment observation, periimplantitis was estimated to occur in $10.7-47.2 \%$ of dental implant patients $^{5}$.

At present, a large majority of commercial dental implants consist of titanium implants, and their surfaces can be modified using several methodologies. However, it is important to note that hydroxyapatite-coated titanium (HA) implants are distinct from non-HA implants in that the implants consist of two different materials (titanium and hydroxyapatite). Hence, it is critical to evaluate the relationship between both implant types and periimplantitis. Some clinical reports have indicated excellent longterm outcomes for both implant types ${ }^{6,7)}$. However, while some investigations of peri-implantitis using non-HA implants have been 

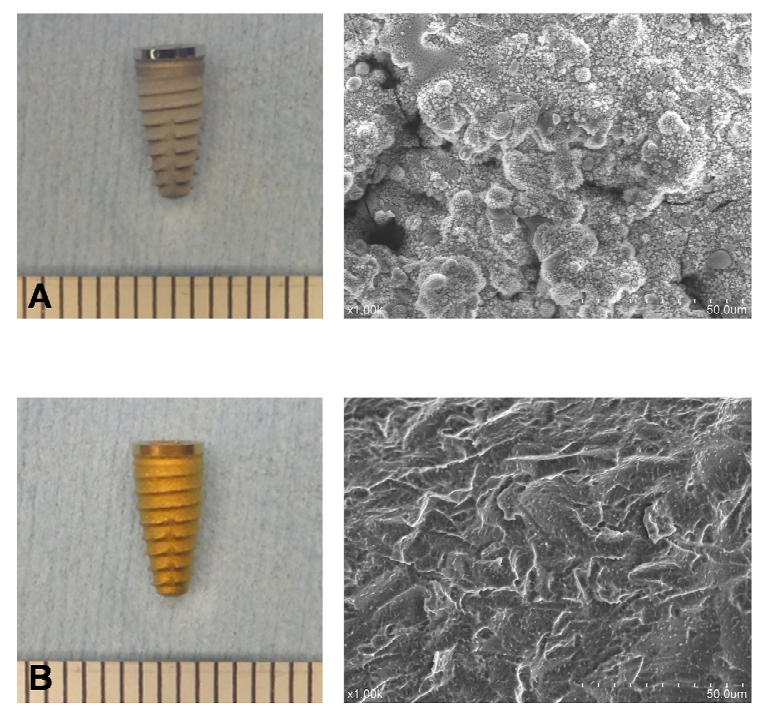

Figure 1. A: Photograph and scanning electron micrograph of the HA implant (HAC-EX; Kyocera Medical, Osaka, Japan); B: Photograph and scanning electron micrograph of the AnO implant (POI-EX; Kyocera Medical, Osaka, Japan). The surface of the HA implant is rougher than that of the AnO implant.

reported $^{8-10)}$, an insufficient number of parallel studies have been conducted using HA implants.

Canine and primate models have been used to study mechanisms involved in the development and progression of periimplantitis $^{11,12)}$. In prior animal models, ligatures constructed of cotton or silk were placed around properly integrated implants and adjusted in the submarginal position, which promoted the buildup of plaque around the neck of the implant. Consequently, inflammatory lesions formed rapidly at the ligature sites, and bone loss occurred ${ }^{13,14)}$. The configurations and sizes of ligature-induced peri-implantitis bone defects in dogs appeared to resemble naturally occurring lesions in humans ${ }^{12}$. However, no differences in the implant types were reported following the animal studies ${ }^{15-}$ 18). Consequently, Renvert et al. concluded that there was no evidence to suggest that implant surface characteristics have significant effects on the induction of peri-implantitis ${ }^{19)}$.

Macroscopic and radiographic evaluations have been performed in previous studies ${ }^{15-17}$. Additionally, histological evaluations were performed in the animal model studies. Further, histomorphometric assessments provide reliable data and can be used to validate radiographic data. However, because of the need for resection, the development and graded progression of periimplantitis in the same individual cannot be observed using this method. As well, the previous studies were not comprehensive with regard to accurate evaluation of the alteration of peri-
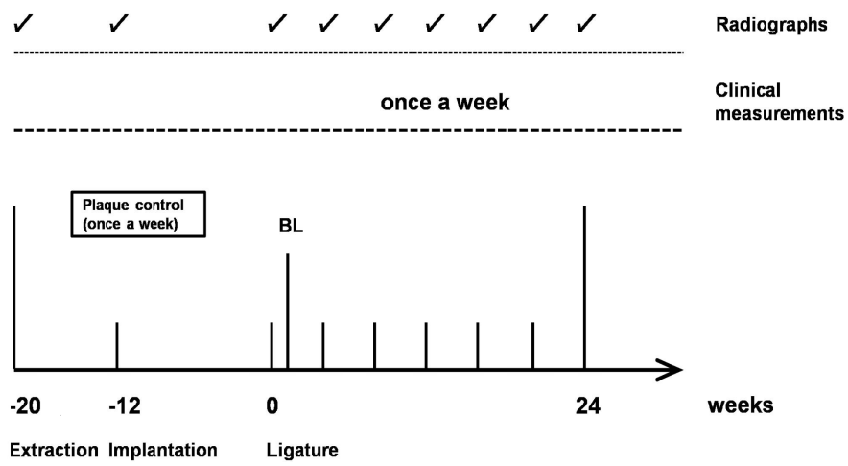

Figure 2. Outline of the current study. One week after ligature placement was considered the baseline (BL). Clinical measurements (mPI, mGI, and PD) were recorded weekly, and radiographs $(>$ ) were acquired monthly until 24 weeks after experimental induction of periimplantitis. mPI, modified plaque index; mGI, modified gingival index; PD, probing depth.

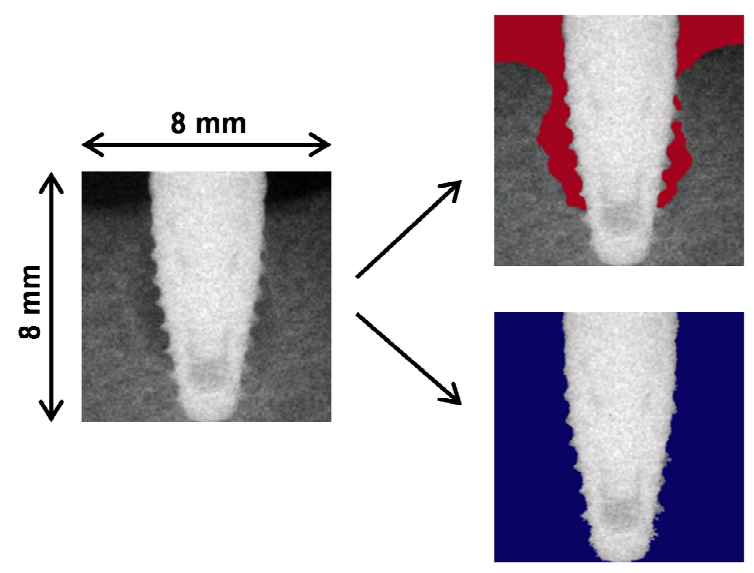

Figure 3. The radiolucent area was expressed as a percentage (\%) of the radiolucent area in the ROI (red)/the ROI minus the area occupied by the implant (blue).

implantitis. Accordingly, it is necessary to develop other mechanisms to define the actual status of peri-implantitis.

Previous studies have established the applicability of multislice computed tomography (MSCT) for preoperative diagnoses in dental implant therapy ${ }^{20,21)}$. Moreover, it is possible to obtain a cross-section of an implant from a multiplanar reconstruction (MPR) image using MSCT. Further, the sagittal plane obtained from an MPR image on the mesiodistal side of the implant was unaffected by metal artifacts in the principle MSCT scan. Hence, this non-invasive inspection method is considered superior to histomorphometry for the chronological evaluation of periimplantitis.

Table 1. Characteristics of the Different Implants used this Study

\begin{tabular}{lcccr}
\hline Implant & Material & Surface & Dimensions & Company \\
\hline HA & Ti-6Al-4V & hydroxyapatite-coated $(20 \mu \mathrm{m})$ & $\varphi 3.7 \mathrm{~mm} \times 8 \mathrm{~mm}$ & HAC-EX, Kyocera Medical, Osaka, Japan \\
AnO & Ti-6Al-4V & anodic oxidized & $\varphi 3.7 \mathrm{~mm} \times 8 \mathrm{~mm}$ & POI-EX, Kyocera Medical, Osaka, Japan \\
\hline
\end{tabular}


Hiroki Nakamura et al.: Peri-Implantitis with Differing Surface Titanium Implants
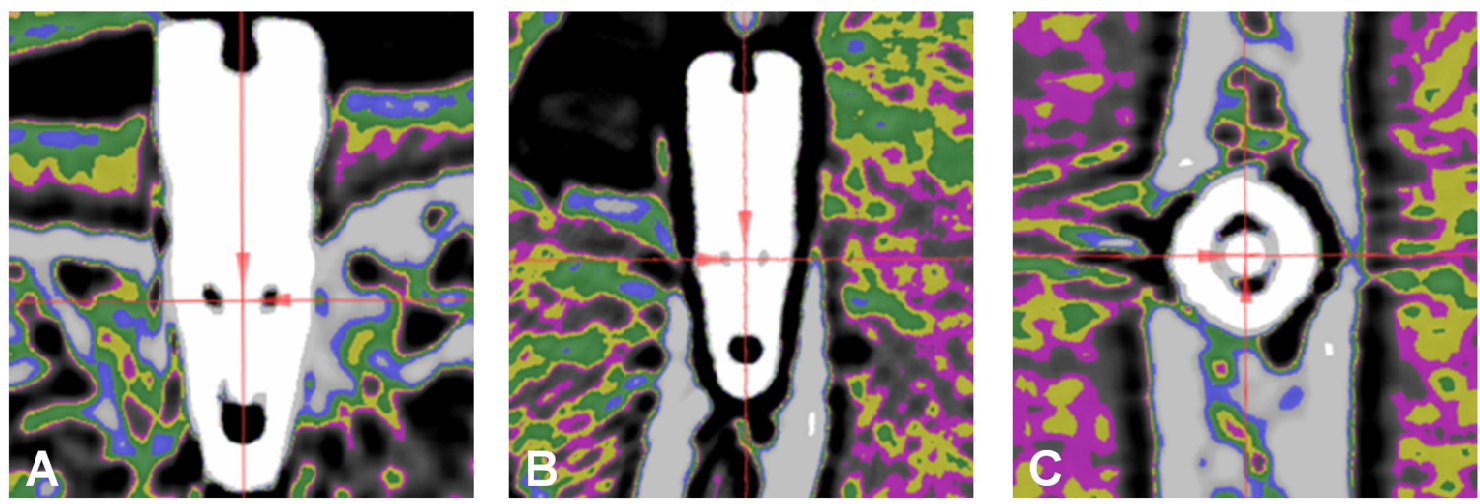

Figure 4. Sagittal (A), coronal (B), and axial (C) reformation of MSCT scans. MPR images were colored based on the classification suggested by Misch ${ }^{23}$. The region with a corresponding CT value less than 0 Hounsfield Unit (HU) was deemed the hyperpermeability area (black and gray).

Accordingly, in the current study, the development and progression of ligature-induced peri-implantitis surrounding HA implants and non-HA implants in dogs were assessed using macroscopic and original radiographic evaluations.

\section{Materials and Methods}

\section{Implants}

Ten titanium alloy (Ti-6Al-4V) dental implants with two different surfaces were used. Five HA implants (HAC-EX; Kyocera Medical, Osaka, Japan; Fig. 1A) and five anodic oxidized (AnO) implants (POI-EX; Kyocera Medical, Osaka, Japan; Fig. 1B) were used. All implants were $8 \mathrm{~mm}$ in length and $3.7 \mathrm{~mm}$ in diameter, and were sandblasted prior to final modification (Table 1).

\section{Animals}

Five adult female beagle dogs were used. At the beginning of the study, the dogs were 2 years old and weighed an average of 10 $\mathrm{kg}$. All experiments were carried out in accordance with the Guidelines stipulated by the National Institutes of Health (NIH) regarding the care and use of animals for experimental procedures, and in accordance with the Principles of Laboratory Animal Care advocated by the Kyoto University Animal Experiment Committee (R-61-10). All surgical and clinical procedures were performed under general anesthesia. Following administration of $0.025 \mathrm{mg} /$ $\mathrm{kg}$ atropine sulfate, the dogs were anesthetized by intramuscular injection of ketamine hydrochloride $(10 \mathrm{mg} / \mathrm{kg})$ and xylazine (1 $\mathrm{mg} / \mathrm{kg})$.

\section{Implant surgery and experimental peri-implantitis}

The outline of the study is presented in Fig. 2. The third and fourth premolars (P3, P4) on each side of the mandible were extracted. After two months, full-thickness flaps were elevated on both sides of the mandible, and each dog had one HA implant and one AnO implant inserted into the center of the respective extraction sites. The implants were placed at the bone level, and a
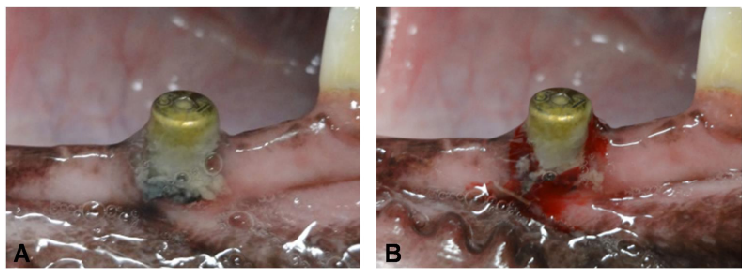

Figure 5. Photographs of ligature-induced peri-implantitis. (A) Plaque formation. (B) Bleeding on probing. Plaque formation resulted in overt signs of mucosal inflammation around the implant.

cover cap was screwed onto the implants. The flaps were sewn with single interrupted sutures to submerge all implants, and after 2 weeks, the sutures were removed. A plaque control program involving dental sheets with $0.05 \%$ chlorhexidine was maintained during the healing period (once a week). Three months later, a healing-cap $(\varphi 4.2 \mathrm{~mm} \times 5 \mathrm{~mm})$ connection was performed. The oral hygiene procedure was stopped after placement of the healing cap, and silk ligature was placed in the submarginal position around the neck of the implants for 24 weeks to induce periimplantitis. To avoid occlusal trauma interference, the feed was soaked in water until the end of the experiment to ensure it was soft prior to feeding.

\section{Macroscopic evaluation}

Macroscopic parameters including modified plaque index (mPI), modified gingival index (mGI), and probing depth (PD) were recorded as previously described ${ }^{22)}$ one week after ligature placement (baseline), and weekly until the end of the experiment. The PD was recorded using a periodontal probe.

\section{Radiographic evaluation}

\section{Dental radiography}

Standardized periapical radiographs were acquired weekly for 24 weeks after ligature placement using a customized film holder, and were transferred to image analysis software (Photoshop, Adobe, CA, USA) to measure the radiolucent area around the 


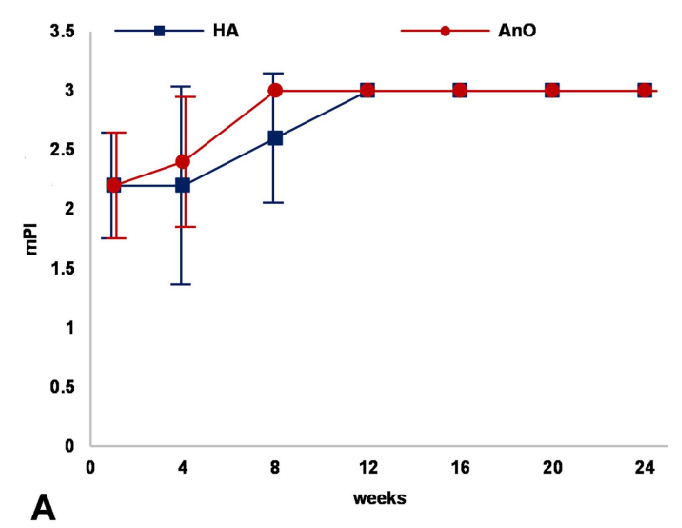

implants. One week after ligature placement was considered the baseline measurement.

Regions of interest (ROI) were defined as the square area $(8$ $\mathrm{mm} \times 8 \mathrm{~mm}$ ) surrounding the implant, and were extracted from radiographic images. The radiolucent area was expressed as a percentage (\%) of the radiolucent area in the ROI/the ROI minus the area occupied by the implant (Fig. 3). Two independent examiners assessed all measurements, and the mean value of the two measurements was used for analysis.

\section{Multislice computed tomography}

MSCT (Aquilion, Toshiba Medical Systems, Tochigi, Japan) scans were performed monthly for 6 months after ligature placement, and the resulting CT data were incorporated into image editing software (Ziostation, Ziosoft, Tokyo, Japan) to construct MPR images.

The dogs were placed on a bed in the gantry, and the mandible was positioned parallel to the horizontal plane of the CT device. After obtaining a scanoscope, the field of view was determined at the table and helical scans were produced around the implants (acquisition collimation: $64 \times 0.5 \mathrm{~mm}$, tube voltage: $120 \mathrm{kV}$, FOV: $120 \mathrm{~mm}$, rot time: $0.5 \mathrm{~s}$, tube current: $200 \mathrm{~mA}$, Eff mAs: 146, pitch: 0.688). A week after ligature placement, the baseline scan was obtained.

Cross-sectional images of the implants were obtained using MPR, and were colored on the basis of the classification established by Misch ${ }^{23)}$. Specifically, the MPR image of the sagittal direction parallel to the longitudinal axis of the implant and the lingual wall of the mandible was created to pass through the central portion of the implant (Fig. 4). The region corresponding to a CT value less than 0 Hounsfield units (HU) was noted as the hyperpermeability area. The images were transferred to image analysis software to measure the hyperpermeability area around the implants. The ROI were established in the same manner as in the dental radiographs. The hyperpermeability area was expressed as a percentage (\%) of the hyperpermeability area in the ROI/the ROI minus the area occupied by the implant.
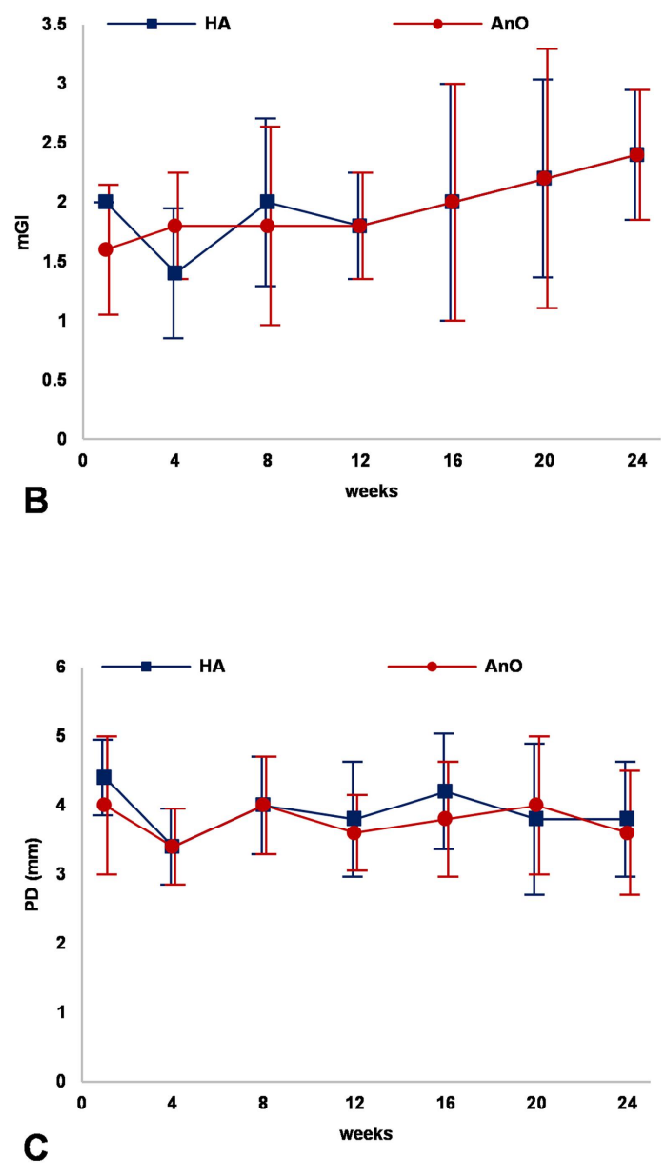

Figure 6. A: Means and standard deviations (SD) of modified plaque index ( $\mathrm{mPI} ; \mathrm{n}=5)$; B: Means and standard deviations (SD) of modified gingival index $(\mathrm{mGI} ; \mathrm{n}=5) ; \mathrm{C}$ : Means and standard deviations (SD) of probing depth (PD; $n=5)$.

\section{Statistical analysis}

Macroscopic and radiographic data were obtained for each implant in each animal, and mean values were calculated $(n=5)$. Macroscopic and radiographic data were compared to baseline values using Dunnett's test, and an unpaired $t$ test was used to calculate differences between the HA and AnO implants. A value of $P<0.05$ was considered to be significant.

\section{Result}

\section{Macroscopic evaluation}

Healing after implant placement was unremarkable at all implant sites. Because there were no outliers, monthly data were used in the graphs. Plaque formation during experimental periimplantitis resulted in overt signs of mucosal inflammation around all implants (Fig. 5). The mean $\mathrm{mPI}$ and $\mathrm{mGI}$ increased during the experimental period for all implants (Fig. 6A, 6B). However, the mean PD did not increase significantly for all implants (Fig. 6C). No statistically significant differences were observed in any macroscopic evaluations of implant surfaces $(P>0.05)$. Further, no mobility was detected in any of the implants following manual 
Hiroki Nakamura et al.: Peri-Implantitis with Differing Surface Titanium Implants
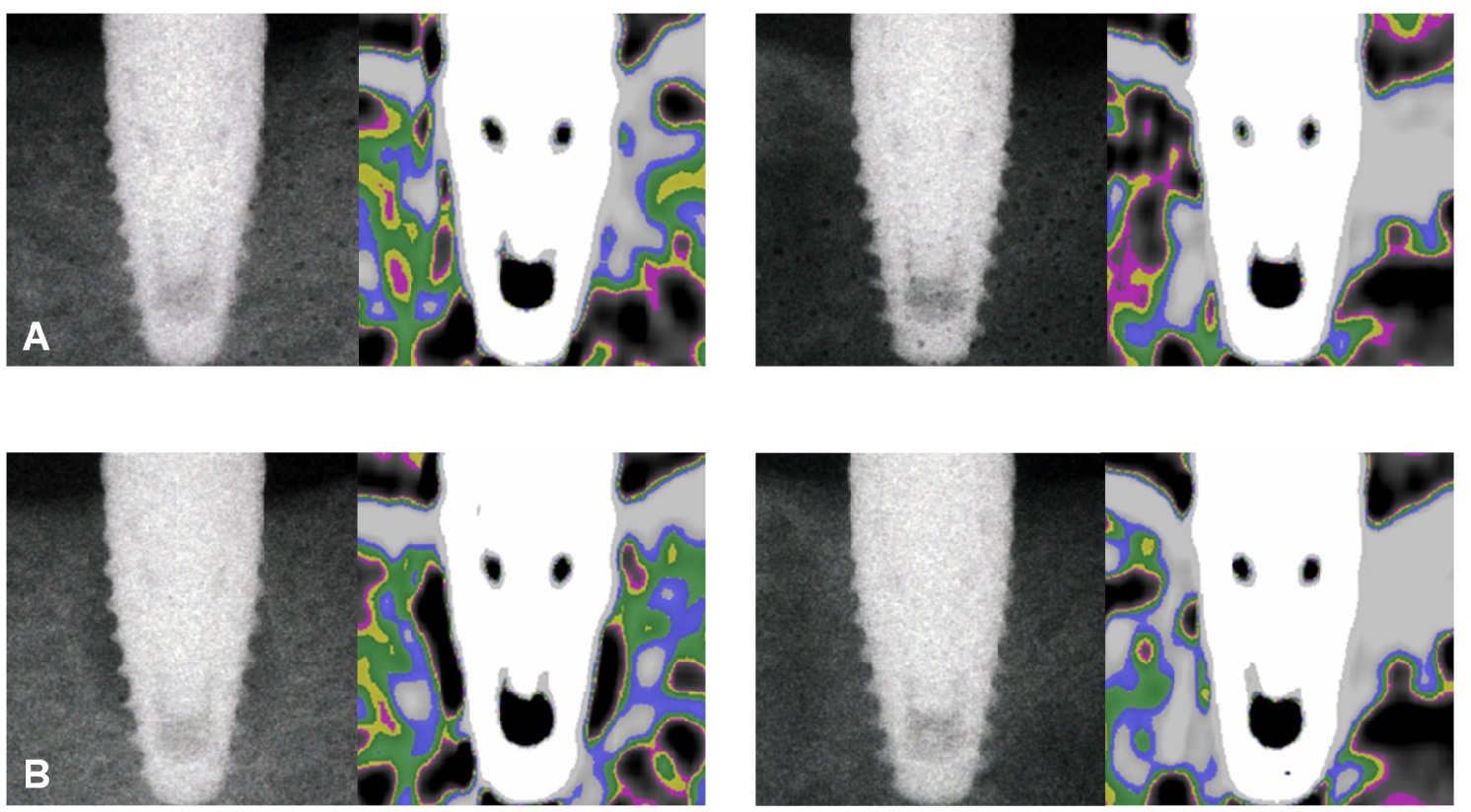

Figure 7. Radiographic images (dental radiography and MSCT) in the ROI from the HA implant (left) and the AnO implant (right) obtained (A) at ligature placement (baseline) and (B) at 24 weeks.

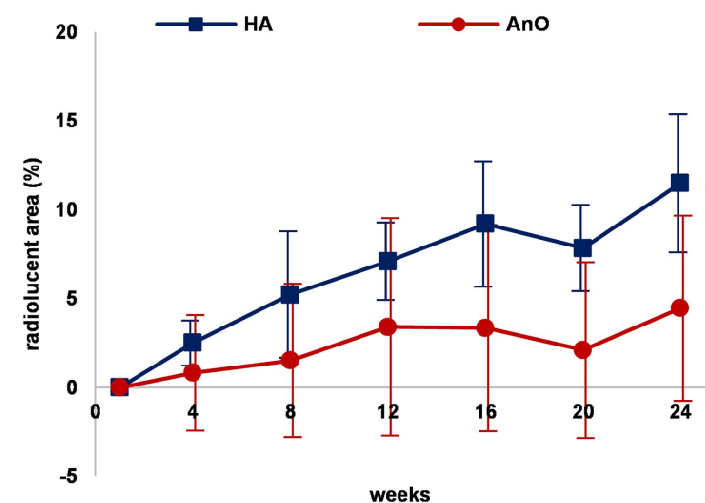

Figure 8. Means and standard deviations (SD) corresponding to alterations in the radiolucent area $(\mathrm{n}=5)$. The radiolucent area of the HA implant increased significantly from baseline $(P<$ 0.05). The radiolucent area of the AnO implant differed significantly from baseline at 24 weeks $(P<0.05)$.

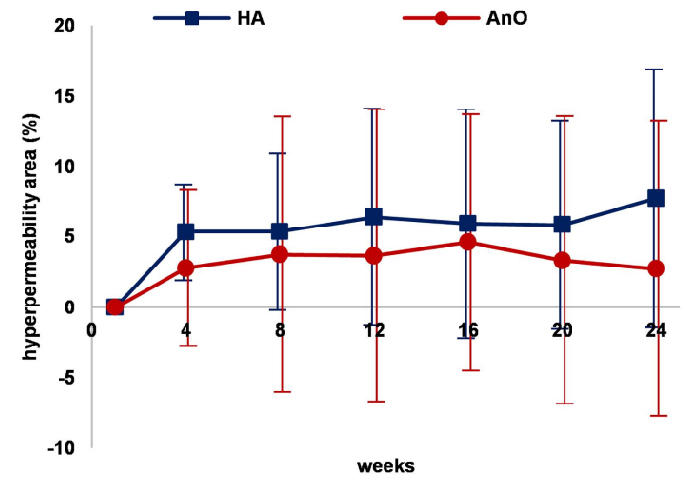

Figure 9. Means and standard deviations (SD) corresponding to alterations in the hyperpermeability area $(n=5)$. The hyperpermeability area of the HA implant differed significantly from baseline at 4 and 24 weeks $(P<0.05)$.

Table 2. Results of Dental Radiographic Evaluations. Radiolucent area alterations (\%) during the Period Following Ligature Placement (20 and 24 weeks - baseline). Mean Values and Standard Deviations (SD; $\mathrm{n}=5$ )

\begin{tabular}{lcc}
\hline Radiolucent area alterations (\%) & HA & AnO \\
\hline 20 weeks - baseline (1 week) & $7.84(2.42)^{* *} \dagger$ & $2.11(4.95)$ \\
24 weeks - baseline (1 week) & $11.5(3.89)^{* *} \dagger$ & $4.46(5.21)^{*}$ \\
\hline
\end{tabular}

*Significant difference from baseline $(P<0.05)$; **Significant difference from baseline $(P<0.01)$; †Significant difference among implant surfaces $(P<0.05)$.

examination during the experimental period.

\section{Radiographic evaluation}

Radiographic images (dental radiography and MSCT) of the
ROI for both the HA implants and the AnO implants obtained at the time of ligature placement (baseline), and at 24 weeks after ligature placement are presented in Fig. 7. 
Table 3. Results of MPR Images of MSCT. Hyperpermeability Area Alteration (\%) during the Period Following Ligature Placement (4 and 24 weeks - baseline). Mean Values and Standard Deviations $(\mathrm{SD} ; \mathrm{n}=5)$

\begin{tabular}{lcr}
\hline Hyperpermeability Area Alterations (\%) & HA & AnO \\
\hline 4 weeks - baseline (1 week) & $5.33(3.39)^{*}$ & $2.79(5.52)$ \\
24 weeks - baseline (1 week) & $7.72(9.16)^{*}$ & $2.74(10.48)$ \\
\hline
\end{tabular}

*Significant difference from baseline $(P<0.05)$

\section{Dental radiography}

The means of the HA implant radiolucent areas increased significantly after ligature placement $(P<0.05)$. The radiolucent areas around the AnO implant differed significantly from baseline at 24 weeks $(P<0.05$; Fig. 8). The means of radiolucent area alterations between baseline and 20 weeks, and between baseline and 24 weeks after ligature placement were $7.84 \% \pm 2.42 \%$ and $11.48 \% \pm 3.89 \%$ for HA implants, respectively, and $2.10 \% \pm$ $4.95 \%$ and $4.46 \% \pm 5.21 \%$ for AnO implants, respectively. The differences in radiolucent area alterations between implant surfaces at 20 and 24 weeks were statistically significant $(P<0.05$; Table 2).

\section{Multislice computed tomography}

Data from two-dimensional MSCT were more likely to be affected by individual differences than those from dental radiography; therefore, the standard deviations were high. Although the hyperpermeability area of the HA implant differed significantly from baseline at 4 and 24 weeks $(P<0.05)$, the mean hyperpermeability area of the AnO implant decreased after 16 weeks. However, no statistically significant differences were observed for the AnO implants during the peri-implantitis induction period ( $P>0.05$; Fig. 9 ). The mean hyperpermeability area alterations between baseline and 24 weeks was $7.72 \% \pm$ $9.16 \%$ for the HA implants, and $2.74 \% \pm 10.48 \%$ for the AnO implants. The differences in hyperpermeability area alterations between implant surfaces at 24 weeks were not statistically significant $(P>0.05$; Table 3$)$.

\section{Discussion}

The current study involved the clinical and radiographic analysis of the development and progression of ligature-induced peri-implantitis surrounding titanium alloy implants with different surface treatments (HA and AnO). During the 24-week active breakdown period following ligature placement, the HA implant appeared to be more susceptible than the AnO implant to periimplantitis. In particular, dental radiography revealed significant differences between the HA and AnO implants.

The same implant and healing-cap sizes were used in the experiment to minimize variance in the study results. Additionally, only one implant was placed on each side of the mandible, which allowed for a sufficient distance from existing teeth. Most prior studies have been performed without considering different implant sizes and distances from existing teeth, notwithstanding the conclusion that all surfaces were equally susceptible to ligature induced peri-implantitis ${ }^{15-18}$. Thus, previous experimental models are thought to be insufficient for the accurate comparison of individual implants, because of the apparent effects from adjacent teeth and other implants.

In our macroscopic evaluations, $\mathrm{mPI}$, and $\mathrm{mGI}$ increased in both implant types during the observation period following ligature placement, which was consistent with the results of a previous study $^{24)}$. Nevertheless, the mean PD for both implant surfaces did not increase compared to baseline, which could be attributed to the procedure used for inducing experimental peri-implantitis. In this study, the healing-cap connection and ligature placement were performed simultaneously, and thus it can be concluded that the inflammation and gingival pockets were induced by second-stage surgery. Consequently, the highest mean PD value was noted at baseline.

In this investigation, cross-sectional images of implants were obtained by MPR using MSCT. Some prior studies have indicated that MSCT was not useful for postoperative dental implant therapy diagnoses, because accurate evaluation of the peri-implant bone after implantation was inhibited by metal artifacts in the CT image $^{25,26)}$. However, Schliephake et al. compared measurements of peri-implant defects in the sagittal and coronal planes using MSCT. Their findings indicated that coronal scans were unreliable for the buccal and lingual sides, whereas the sagittal views revealed good correlation with the gold standard ${ }^{27}$. Accordingly, the use of MSCT was substituted for histological evaluation of periimplant bone.

In our radiographic assessments, bone loss around the implant was evaluated according to the area (\%) of all radiographic images. Previous studies have investigated bone loss on radiographic findings by evaluating marginal bone level (MBL) ${ }^{15,28}$. However, in the current experiment, discontinuous bone loss was detected around the implant area. Further, the bone loss expanded not only from the marginal portion, but also from deep implant surfaces, and thus consideration should be given to the conventional method when evaluating the deep site of the implant.

The HA implant exhibited a significant increase in the radiolucent area after 24 weeks of induced peri-implantitis when compared to the AnO implant. However, some previous reports 
Hiroki Nakamura et al.: Peri-Implantitis with Differing Surface Titanium Implants

performed using similar protocols have indicated that there were no significant differences between HA and non-HA implants in the development and progression of peri-implantitis for 6 months ${ }^{17,18)}$. In fact, in these reports HA implants were associated with a greater degree of bone loss than non-HA implants. A potential explanation for the lack of significant differences identified could be the small sample size. Nonetheless, a more detailed assessment of peri-implantitis was achieved following our original radiographic evaluations, and the influence of periimplantitis induced by the HA implant was more accurately described.

The mean radiolucent areas of the $\mathrm{HA}$ and $\mathrm{AnO}$ implants increased significantly between baseline and 24 weeks after ligature placement. However, the HA implant exhibited a significant increase in the initial phase of the peri-implantitis induction, while the $\mathrm{AnO}$ implant exhibited a significant increase in the last phase ( 24 weeks after baseline). In addition, the mean hyperpermeability area of the HA implant increased significantly between baseline and 24 weeks after ligature placement, while the mean hyperpermeability area of the AnO implant decreased after 16 weeks. Alterations in the hyperpermeability area during the peri-implantitis induction period were not statistically significant. Our results in this regard differed from those of previous studies. Martins et al. evaluated experimental periimplantitis associated with different commercially available implants (commercially pure titanium, titanium plasma-sprayed, HA, and acid-etched). The macroscopic and radiographic data collected during the initial phase (up to 60 days after ligature placement) revealed rapid and severe peri-implant tissue breakdown in all types of implants ${ }^{15,28}$. The differences suggest that the AnO implants possess greater resistance to peri-implantitis than the HA implants in experimental situations under similar size and implantation conditions.

In this study, our macroscopic results were not consistent with our radiographic results. Prior clinical studies have indicated that PD was deeper in implants that presented with radiographic bone loss compared with implants that had no associated bone loss ${ }^{29,30}$. However, at present, no studies have investigated PD of implants with associated peri-implantitis, and thus the relationship between $\mathrm{PD}$ and the peri-implant bone level remains speculative. Serino et al. reported that measurements of peri-implantitis associated with prosthetic reconstruction implants generated in situ should be interpreted cautiously with regard to their ability to determine the severity of bone loss at implants ${ }^{31)}$. Qualitatively, radiographic evaluation revealed that $\mathrm{PD}$ tended to be proportional to MBL, because the probe tip penetrated to a position closer to the alveolar bone crest in sites with peri-implant disease ${ }^{32,33)}$. However, no relationship existed between macroscopic and radiographic evaluations in this experiment, because the bone loss appeared around the deep site of the implant, and was not detectable using a periodontal probe. Consequently, bone loss was only assessed using radiography. The results of the current study emphasized the importance of radiographic analysis, and suggested that the conventional assessment of bone loss should be reconsidered.

A limitation of this study included the use of plaque-induced peri-implantitis without occlusion to simplify the evaluation. However, occlusal overload may lead to bone loss in the presence of dental plaque ${ }^{34,35)}$. This model was not suitable for the evaluation of the influence of clinical occlusion on development of periimplantitis. Furthermore, the results should be interpreted cautiously due to the small sample size, the short evaluation period, and impossibility of the histological evaluation.

In the current study, the HA implant exhibited larger radiolucent and hyperpermeability areas over the course of the study than the AnO implant. Additionally, the progression of bone loss associated with the HA implant appeared around the deep site of the implant, which was in contrast to the localized bone loss apparent with the AnO implant.

Our original evaluations, which assessed bone loss according to the area $(\%)$ of all radiographic images, were effective for comparing the development and graded progression of periimplantitis between the implant types in the same individual. Further, our results indicate that the resistance of the HA implant to peri-implantitis is inferior to that of the $\mathrm{AnO}$ implant.

\section{Acknowledgments}

The authors also thank Kyocera Medical Corporation, Japan, for supplying all of the implants used in this study.

\section{References}

1. Lekholm U, Gunne J, Henry P, Higuchi K, Lindén U, Bergström C and van Steenberghe D. Survival of the Branemark implant in partially edentulous jaws: A 10-year prospective multicenter study. Int J Oral Maxillofac Implants 14: 639-645, 1998

2. Rosenberg ES, Cho S-C, Elian N, Jalbout ZN, Froum S and Evian CI. A comparison of characteristics of implant failure and survival in periodontally compromised and periodontally healthy patients: A clinical report. Int J Oral Maxillofac Implants 19: 873-879, 2003

3. Snauwaert K, Duyck J, van Steenberghe D, Quirynen M and Naert I. Time dependent failure rate and marginal bone loss of implant supported prostheses: A 15-year follow-up study. Clin Oral Invest 4: 13-20, 2000

4. Zitzmann NU and Berglundh T. Definition and prevalence of peri-implant diseases. J Clin Periodontol 35: 286-291, 2008

5. de Waal Y, van Winkelhoff AJ, Meijer HJ, Raghoebar GM and Winkel EG. Differences in peri-implant conditions between fully and partially edentulous subjects: A systematic 
review. J Clin Periodontol 40: 266-286, 2013

6. Schwartz-Arad D, Mardinger O, Levin L, Kozlovsky A and Hirshberg A. Marginal bone loss pattern around hydroxyapatite-coated versus commercially pure titanium implants after up to 12 years of follow-up. Int J Oral Maxillofac Implants 20: 238-244, 2004

7. McGlumphy EA, Peterson LJ, Larsen PE and Jeffcoat MK. Prospective study of 429 hydroxyapatite-coated cylindric omniloc implants placed in 121 patients. Int J Oral Maxillofac Implants 18: 82-92, 2002

8. Wennström JL, Ekestubbe A, Gröndahl K, Karlsson S and Lindhe J. Oral rehabilitation with implant-supported fixed partial dentures in periodontitis-susceptible subjects. J Clin Periodontol 31: 713-724, 2004

9. Zetterqvist L, Feldman S, Rotter B, Vincenzi G, Wennström J L, Chierico A, Stach R M and Kenealy J N. A prospective, multicenter, randomized-controlled 5-year study of hybrid and fully etched implants for the incidence of periimplantitis. J Periodontol 81: 493-501, 2010

10. Åstrand P, Engquist B, Anzén B, Bergendal T, Hallman M, Karlsson U, Kvint S, Lysell L and Rundcranz T. A threeyear follow-up report of a comparative study of ITI Dental Implants ${ }^{\circledR}$ and Brånemark System ${ }^{\circledR}$ implants in the treatment of the partially edentulous maxilla. Clin Implant Dent Relat Res 6: 130-141, 2004

11. Lindhe J, Berglundh T, Ericsson I, Liljenberg B and Marinello C. Experimental breakdown of peri-implant and periodontal tissues. A study in the beagle dog. Clin Oral Implants Res 3: 9-16, 1992

12. Schwarz F, Herten M, Sager M, Bieling K, Sculean A and Becker J. Comparison of naturally occurring and ligatureinduced peri-implantitis bone defects in humans and dogs. Clin Oral Implants Res 18: 161-170, 2007

13. Marinello C, Berglundh T, Ericsson I, Klinge B, Glantz P and Lindhe J. Resolution of ligature-induced peri-implantitis lesions in the dog. J Clin Periodontol 22: 475-479, 1995

14. Persson LG, Araújo MG, Berglundh T, Gröndahl K and Lindhe J. Resolution of peri-implantitis following treatment. An experimental study in the dog. Clin Oral Implants Res 10: 195-203, 1999

15. Martins MC, Abi-Rached R, Shibli JA, Araujo M and Marcantonio Jr E. Experimental peri-implant tissue breakdown around different dental implant surfaces: Clinical and radiographic evaluation in dogs. Int J Oral Maxillofac Implants 19: 839-848, 2003

16. Shibli JA, Martins MC, Lotufo R and Marcantonio JrE. Microbiologic and radiographic analysis of ligature-induced peri-implantitis with different dental implant surfaces. Int J Oral Maxillofac Implants 18: 383-390, 2002

17. Tillmanns H, Hermann JS, Cagna DR, Burgess AV and Meffert
RM. Evaluation of three different dental implants in ligatureinduced peri-implantitis in the beagle dog. Part I. Clinical evaluation. Int J Oral Maxillofac Implants 12: 611-620, 1996

18. Tillmanns HW, Hermann JS, Tiffee JC, Burgess AV and Meffert RM. Evaluation of three different dental implants in ligature-induced peri-implantitis in the beagle dog. Part II. Histology and microbiology. Int J Oral Maxillofac Implants 13: 59-68, 1998

19. Renvert S, Polyzois I and Claffey N. How do implant surface characteristics influence peri-implant disease? J Clin Periodontol 38: 214-222, 2011

20. Naitoh M, Katsumata A, Kubota Y and Ariji E. Assessment of three-dimensional X-ray images: Reconstruction from conventional tomograms, compact computerized tomography images, and multislice helical computerized tomography images. J Oral Implantol 31: 234-241, 2005

21. Naitoh M, Katsumata A, Nohara E, Ohsaki C and Ariji E. Measurement accuracy of reconstructed 2-D images obtained by multi-slice helical computed tomography. Clin Oral Implants Res 15: 570-574, 2004

22. Mombelli A, Oosten M, Schürch E and Lang N. The microbiota associated with successful or failing osseointegrated titanium implants. Oral Microbiol Immunol 2: 145-151, 1987

23. Misch CE. Density of bone: effect on treatment plans, surgical approach, healing, and progressive bone loading. Int J Oral Implantol 6 (2): 23-31, 1990

24. Zechner W, Kneissel M, Kim S, Ulm C, Watzek G and Plenk H. Histomorphometrical and clinical comparison of submerged and nonsubmerged implants subjected to experimental peri-implantitis in dogs. Clin Oral Implants Res 15: 23-33, 2004

25. Barrett JF and Keat N. Artifacts in CT: Recognition and avoidance 1. Radiographics 24: 1679-1691, 2004

26. Dalal T, Kalra M K, Rizzo S M, Schmidt B, Suess C, Flohr T, Blake M A and Saini S. Metallic Prosthesis: Technique to avoid increase in $\mathrm{CT}$ radiation dose with automatic tube current modulation in a phantom and patients 1 . Radiology 236: 671-675, 2005

27. Schliephake H, Wichmann M, Donnerstag F and Vogt S. Imaging of periimplant bone levels of implants with buccal bone defects. Clin Oral Implants Res 14: 193-200, 2003

28. Martins MC, Shibli JA, Abi-Rached RSG and Marcantonio Jr E. Progression of experimental chronic peri-implantitis in dogs: Clinical and radiographic evaluation. J Periodontol 76: 1367-1373, 2005

29. Fransson C, Wennström J and Berglundh T. Clinical characteristics at implants with a history of progressive bone loss. Clin Oral Implants Res 19: 142-147, 2008

30. Hultin M, Gustafsson A, Hallström H, Johansson LÅ, Ekfeldt 
Hiroki Nakamura et al.: Peri-Implantitis with Differing Surface Titanium Implants

A and Klinge B. Microbiological findings and host response in patients with peri-implantitis. Clin Oral Implants Res 13: 349-358, 2002

31. Serino G, Turri A and Lang NP. Probing at implants with peri-implantitis and its relation to clinical peri-implant bone loss. Clin Oral Implants Res 24: 91-95, 2013

32. Abrahamsson I and Soldini C. Probe penetration in periodontal and peri-implant tissues: An experimental study in the beagle dog. Clin Oral Implants Res 17: 601-605, 2006

33. Schou S, Holmstrup P, Stoltze K, Hjørting-Hansen E, Fiehn
NE and Skovgaard LT. Probing around implants and teeth with healthy or inflamed peri-implant mucosa/gingiva. Clin Oral Implants Res 13: 113-126, 2002

34. Chambrone L, Chambrone LA and Lima LA. Effects of occlusal overload on peri-implant tissue health: A systematic review of animal-model studies. J Periodontol 81: 13671378,2010

35. Naert I, Duyck J and Vandamme K. Occlusal overload and bone/implant loss. Clin Oral Implants Res 23: 95-107, 2012 
J.Hard Tissue Biology Vol. 24(4):347 -356, 2015 